\title{
Small-Molecule Bulk Heterojunctions: Distinguishing Between Effects of Energy Offsets and Molecular Packing on Optoelectronic Properties
}

\author{
K. Paudel ${ }^{\dagger}$ B. Johnson, ${ }^{\dagger}$ A. Neunzert, ${ }^{\dagger}$ M. Thieme, ${ }^{\dagger}$ B. Purushothaman, ${ }^{\ddagger}$ M. M. Payne, ${ }^{\ddagger}$ J. E. Anthony, ${ }^{\ddagger}$ \\ and O. Ostroverkhova ${ }^{\S, *}$
}

${ }^{\dagger}$ Department of Physics Oregon State University, Corvallis, Oregon 97331, United States

${ }^{\ddagger}$ Department of Chemistry, University of Kentucky, Lexington, Kentucky 40506, United States

${ }^{\S}$ Department of Physics, Oregon State University, Corvallis, Oregon 97331, United States

\section{Supporting Information}

ABSTRACT: We report on the dependence of time-resolved photoluminescence (PL) and photocurrent in small-molecule bulk heterojunctions on the donoracceptor (D/A) LUMO offset, D/A separation, and acceptor domain structure. We chose a high-performance functionalized fluorinated anthradithiophene (ADT) derivative, ADT-TES-F, as the donor and two other fluorinated ADT derivatives, ADT-R-F (where $\mathrm{R}$ is a variable side group), as well as two functionalized fluorinated pentacene $(\mathrm{Pn})$ derivatives, Pn-R-F8, as acceptors. The choice of ADT and Pn acceptors enabled us to separate the effects of the D/A LUMO offset, which was approximately zero in the case of ADT acceptors and $\sim 0.55 \mathrm{eV}$ in the case of $\mathrm{Pn}$ acceptors, from those of molecular packing on the optoelectronic properties. The acceptor side groups $\mathrm{R}$ were chosen based on (i) packing motifs in the solid state and (ii) size, to achieve different D/A separations at the D/A interface. Addition of an ADT-R-F acceptor to the ADT-TES-F donor introduced disorder, which resulted in increased PL emission, depending on the acceptor's packing motif, and in reduced photocurrents. In ADT-TES-F/Pn-R-F8 films, charge transfer from ADT-TES-F to Pn-R-F8 was observed with an acceptor packing-dependent formation of an exciplex, which dissociated under applied electric field, contributing to charge carrier photogeneration. However, this contribution was not sufficient to compensate for a photocurrent reduction due to an increased disorder at Pn-R-F8 concentrations of $7 \mathrm{wt} \%$ and above, regardless of the acceptor's R-groups and packing motifs.

\section{INTRODUCTION}

Organic donor-acceptor (D/A) composites are of interest for a variety of optoelectronic applications due to their low cost, relatively simple fabrication, and tunable properties. ${ }^{1-7}$ Most of these applications rely on the photoconductive properties of materials, and therefore it is important to understand charge carrier photogeneration and transport in organic D/A materials and how they are affected by the choice of the donor (D) and acceptor (A) molecules. A considerable research effort has been focused on charge photogeneration and subsequent transport in polymer-based $\mathrm{D} / \mathrm{A}$ bulk heterojunctions $(\mathrm{BHJs}){ }^{8-11}$ while fewer studies aimed to determine physical mechanisms behind optoelectronic properties of small-molecule D/A systems. ${ }^{12-18}$ It has been established that the key factors that dramatically affect charge photogeneration at the $\mathrm{D} / \mathrm{A}$ interfaces are the energy offsets between the LUMO or HOMO energy levels of the $\mathrm{D}$ and A molecules ${ }^{19,20}$ and the molecular packing of the $\mathrm{D}$ and $\mathrm{A}^{18,21,22}$ The former determines the driving force for the electron or hole transfer, ${ }^{19,23-26}$ while the latter affects the separation between the $\mathrm{D}$ and $\mathrm{A}$ molecules at the $\mathrm{D} / \mathrm{A}$ interface and the morphology of the D/A interface, ${ }^{27}$ as well as determines $\mathrm{D}$ and A domain structures. ${ }^{28,18}$ More precisely, D/
A separation at the $\mathrm{D} / \mathrm{A}$ interface significantly affects both the efficiency of charge transfer (CT) between D and A and of CT exciton dissociation, ${ }^{18,21,29-32}$ whereas the $\mathrm{D}$ and $\mathrm{A}$ domain structures determine the hole and electron mobilities, respectively, exciton diffusion lengths, and the area of the D/ A interface. ${ }^{28,33}$ For example, it has been established that interdigitated networks of $\mathrm{D}$ and $\mathrm{A}$ domains, with a large $\mathrm{D} / \mathrm{A}$ interfacial area, large $\mathrm{D} / \mathrm{A}$ separation, and high intradomain order are desirable for organic BHJ solar cells. ${ }^{21,34-37}$ However, given the multitude of all of the factors above, it is challenging to evaluate their separate contributions to the device characteristics and to identify a dominant performance-limiting factor, which is necessary for the development of improved D and A materials. In this paper, we seek to separately study effects of $\mathrm{D} / \mathrm{A}$ energy offsets, of D/A separation at the D/A interface, and of acceptor domain structures on exciton and charge carrier dynamics in small-molecule D/A composites.

Received: September 17, 2013

Revised: October 18, 2013

Published: October 24, 2013 
In particular, we examined $\mathrm{D} / \mathrm{A}$ composites with a fluorinated anthradithiophene (ADT) derivative functionalized with triethylsilylethylyl (TES) side groups, ADT-TES-F, as the donor (Figure 1). ADT-TES-F is a high-performance organic

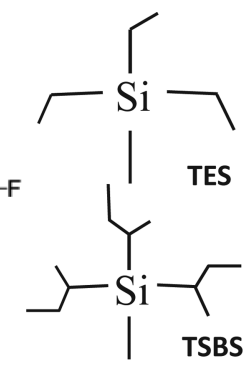

$R=T E S, T I P S, T S B S$

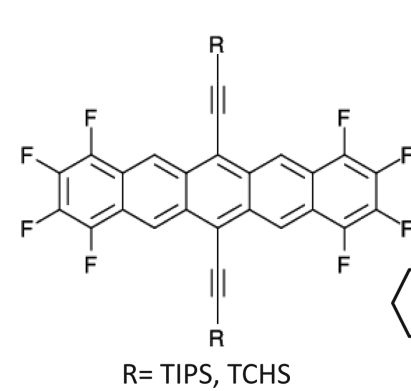

$\mathrm{S}_{\mathrm{Si}} \gamma_{\text {TIPS }}$

$\mathrm{R}=\mathrm{TIPS}, \mathrm{TCHS}$

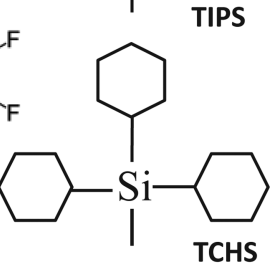

Figure 1. Molecular structures of the ADT-R-F (top; $\mathrm{R}=\mathrm{TES}$ (donor) and TIPS or TSBS (acceptors)) and Pn-R-F8 derivatives (bottom; R = TIPS or TCHS) used in our studies (left). The side groups $\mathrm{R}$, where $\mathrm{R}=\mathrm{TES}$, TIPS, TSBS, and TCHS, are also shown (right).

semiconductor that forms solution-deposited crystalline films exhibiting thin-film transistor (TFT) hole mobilities of $>1.5$ $\mathrm{cm}^{2} /(\mathrm{Vs}) .^{38}$ The acceptors (Figure 1) were chosen given the following considerations.

(1) In order to separate effects of D/A energy offsets from those of molecular packing on optoelectronic properties, two functionalized $\mathrm{ADT}$ and two pentacene $(\mathrm{Pn})$ derivatives (ADTR-F and Pn-R-F8, respectively, where $\mathrm{R}$ is a variable side group, Figure 1) were selected as acceptors. The ADT-R-F derivatives, ADT-TIPS-F (TIPS = triisopropylsilylethynyl) and ADTTSBS-F (trisec-butylsilylethynyl), used here as "acceptor" molecules, have HOMO and LUMO energies, as well as optical properties, similar to those of the ADT-TES-F donor, ${ }^{39}$ so that the D/A LUMO offset is approximately zero. In contrast, in ADT-TES-F/Pn-R-F8 composites, the D/A LUMO offset is about $0.55 \mathrm{eV}$ (Table 1$){ }^{18}$ which has been predicted by Monte Carlo simulations to yield optimal small-molecule solar cell performance. ${ }^{40}$

(2) In order to study effects of the D/A separation on optoelectronic properties, for both $\mathrm{ADT}$ and Pn acceptors we selected derivatives with R-groups of different sizes. In particular, the TIPS group in ADT-TIPS-F and Pn-TIPS-F8 is considerably smaller than the TSBS group in ADT-TSBS-F or the TCHS group (TCHS = tricyclohexylsilylethynyl) in PnTCHS-F8 (Figure 1 and Table 1). Thus, a considerably smaller $\mathrm{D} / \mathrm{A}$ separation is expected in the $\mathrm{D} / \mathrm{A}$ composites with acceptors containing TIPS groups as compared to those with TSBS or TCHS groups. ${ }^{18}$

(3) It has been previously established that the choice of side groups $\mathrm{R}$ in our materials determines molecular packing in the solid state. ${ }^{41}$ In order to study effects of acceptor domain structure on the optoelectronic properties, for both $\mathrm{ADT}$ and Pn acceptors we selected R-groups that promote dramatically different $\pi$-stacking motifs (Table 1 ). In particular, ADT-TIPS$\mathrm{F}$ and Pn-TIPS-F8 molecules exhibit a two-dimensional (2D) "brick-work" packing, similar to that of the ADT-TES-F donor. $^{42,22}$ In contrast, ADT-TSBS-F and Pn-TCHS-F8 molecules pack in a one-dimensional (1D) "sandwichherringbone" structure. ${ }^{22,43}$

In all $\mathrm{D} / \mathrm{A}$ composites, we measured time-resolved and steady-state photoluminescence (PL) and photocurrent, depending on the acceptor and on the acceptor concentration.

\section{EXPERIMENTAL SECTION}

2.1. Materials. Molecular structures of materials used in our study are shown in Figure 1, and estimates of their HOMO and LUMO energies measured using differential pulse voltammetry $^{39}$ are included in Table 1 . We use the volume of side groups $\mathrm{R}$ (Table 1), obtained from crystallographic data, as a relative measure of the $\mathrm{D}$ and $\mathrm{A}$ separation at the D/A interfaces, ${ }^{18}$ such that we expect larger D/A separations in D/A composites with acceptors containing TCHS and TSBS R-groups, as compared to those with TIPS R-groups.

Detailed crystallographic information on the ADT and Pn derivatives used in our studies was reported elsewhere. ${ }^{42-45}$ Briefly, the ADT-TES-F donor and ADT-TIPS-F and Pn-TIPSF8 acceptors assume a $2 \mathrm{D}$ "brick-work" $\pi$-stacking, which is a packing motif favorable for TFTs. ${ }^{43}$ The ADT-TSBS-F and PnTCHS-F8 derivatives pack in a 1D "sandwich-herringbone" structure, which has been shown to be favorable for BHJ solar cells with a P3HT polymeric donor and functionalized Pn acceptors. $^{22}$

All three ADT derivatives have been previously investigated in field effect transistors (FETs). The best hole mobilities observed were $>1.5 \mathrm{~cm}^{2} /(\mathrm{Vs})\left(6 \mathrm{~cm}^{2} /(\mathrm{Vs})\right)$ in spin-cast ADTTES-F films (single crystals), ${ }^{46-48} 0.1 \mathrm{~cm}^{2} /(\mathrm{Vs})$ in ADT-TIPS$\mathrm{F}$ single crystals, ${ }^{42}$ and $0.001 \mathrm{~cm}^{2} /(\mathrm{Vs})$ in drop-cast $\mathrm{ADT}$ TSBS-F films; ${ }^{43}$ such pronounced differences in charge carrier mobilities are largely due to differences in film structure and

Table 1. Electrochemical, Optical, and Crystallographic Properties of ADT-R-F and Pn-R-F8 Molecules Used in Our Studies

\begin{tabular}{|c|c|c|c|c|c|}
\hline material & HOMO (LUMO) $(\mathrm{eV})^{a}$ & $\lambda_{\mathrm{abs}}\left(\lambda_{\mathrm{PL}}\right)(\mathrm{nm})$ & $\lambda_{\mathrm{abs}}^{\text {film }}\left(\lambda_{\mathrm{PL}}^{\text {film }}\right)(\mathrm{nm})^{d}$ & volume of R-group $\left(\AA^{3}\right)^{e}$ & packing \\
\hline ADT-TES-F & $-5.35(-3.05)$ & $528(536)^{b}$ & $552(582)$ & 204 & 2D "brick-work" \\
\hline ADT-TIPS-F & $-5.34(-3.05)$ & $528(536)^{b}$ & $538(548)$ & 278.5 & 2D "brick-work" \\
\hline ADT-TSBS-F & $-5.35(-3.05)$ & $528(536)^{b}$ & $532(552)$ & 353 & 1D "sandwich-herringbone" \\
\hline Pn-TIPS-F8 & $-5.55(-3.60)$ & $635(642)^{c}$ & $646(664)$ & 278.5 & 2D "brick-work" \\
\hline Pn-TCHS-F8 & $-5.54(-3.59)$ & $635(642)^{c}$ & $641(651)$ & 469.3 & 1D "sandwich-herringbone" \\
\hline
\end{tabular}

${ }^{a}$ Measured by differential pulse voltammetry. ${ }^{39,45}{ }^{b}$ Measured in toluene. ${ }^{c}$ Measured in chlorobenzene. ${ }^{d}$ Measured in thin films spin-cast on PFBTtreated substrates as described in Section $2.3{ }^{e}$ Calculated from crystallographic data. 
morphology and crystal quality. ${ }^{39}$ It has been established that all three ADT derivatives, when deposited from solution, form polycrystalline films in which the crystallites are oriented such that the $a-b$ plane, characterized by the strongest $\pi$-overlap and highest charge carrier mobility, is in the plane of the substrate. ${ }^{49,47,42}$

Pn-TIPS-F8 films have also been explored in FET structures, and in vapor-deposited films ambipolar TFT mobilities of up to $0.33 \mathrm{~cm}^{2} /$ (Vs) were obtained. ${ }^{44,50}$ However, solution deposited films of this derivative showed TFT hole mobilities of only $10^{-5} \mathrm{~cm}^{2} /(\mathrm{Vs})$ due to poor film morphology. ${ }^{45}$ To the best of our knowledge, no TFT studies of Pn-TCHS-F8 films have been reported.

Detailed analysis of optical absorption and PL emission spectra of ADT-R-F molecules in solution and in pristine thin films can be found elsewhere. ${ }^{39,51-54}$ Briefly, it has been established that the spectra of isolated ADT-R-F molecules (i.e., in solutions or dispersed at low concentrations in solid matrices) are not affected by the side group R. In contrast, both the absorption and the PL spectra of pristine ADT-R-F films exhibit differences in the wavelengths of absorption and PL maxima (Table 1, Figure S1) and in Huang-Rhys factors, ${ }^{39,51,53}$ dependent on the molecular packing in the film dictated by the side groups $\mathrm{R}^{55}$ The absorption spectra of functionalized ADT and Pn derivatives are red-shifted in films as compared to those of solutions by an amount which depends on the molecular packing and is also related to the strength of intermolecular interactions which promote exciton delocalization. $^{51,56}$ Of all ADT-R-F derivatives used in our studies, the red shift was highest in pristine ADT-TES-F films, which suggests the highest exciton delocalization is in these films, followed by that in pristine ADT-TIPS-F and ADT-TSBS-F films (Table 1). In pristine Pn-R-F8 films, only low red shifts were observed (Table 1, Figure S1), which could be related to poor film crystallinity. ${ }^{56}$

2.2. Sample Preparation. To prepare D/A films, we used $30 \mathrm{mM}$ solutions containing a mixture of ADT-TES-F as the donor and ADT-TIPS-F, ADT-TSBS-F, Pn-TIPS-F8, or PnTCHS-F8 as an acceptor in appropriate concentrations in chlorobenzene. For each D/A combination, the acceptor concentration was varied between 2 and $10 \mathrm{wt} \%$, and $10 \mu \mathrm{L}$ solutions of each D/A mixture were spun at $3000 \mathrm{rpm}$ on a glass substrate patterned with $\mathrm{Cr} / \mathrm{Au}(5 \mathrm{~nm} / 50 \mathrm{~nm})$ interdigitated electrodes pretreated with PFBT. The PFBT treatment had been found to facilitate crystallization in fluorinated $\mathrm{ADT}$ derivatives and to reduce the injection barrier on $\mathrm{Au}$ by increasing its work function, ${ }^{57}$ resulting in goodquality polycrystalline films with considerably improved TFT charge carrier mobilities. ${ }^{38}$ The interdigitated electrodes consisted of 10 pairs of $1 \mathrm{~mm}$ long and $25 \mu \mathrm{m}$ wide fingers, with a gap of $25 \mu \mathrm{m}$ between the fingers. Pristine ADT-TES-F donor films were also prepared using the same protocol. Such preparation method yielded polycrystalline films as confirmed by X-ray diffraction ${ }^{43,49}$ and optical imaging. Formation of micrometer-sized crystalline acceptor domains could be observed at acceptor concentrations above 5\%, as shown in Figure S2.

2.3. Optical Absorption and PL Measurements. Optical absorption spectra were taken using an Ocean Optics LS-1 tungsten halogen lamp and an Ocean Optics USB4000 spectrometer. The photoluminescence (PL) was excited by a $532 \mathrm{~nm}$ frequency-doubled $\mathrm{Nd}: \mathrm{YVO}_{4}$ laser from Coherent, Inc. PL spectra were then collected using an Ocean Optics
USB2000 spectrometer in a $45^{\circ}$ reflection geometry, containing an HQ535LP filter from Chroma Tech.

For PL lifetime measurements, the samples were excited with a $355 \mathrm{~nm} 500$ ps laser (Q-switched, frequency-tripled Nd:YAG from Nanolase, Inc.). Signals were acquired by a timecorrelated single-photon counting (TCSPC) board (PicoQuant Time Harp 200) through a single-photon avalanche photodiode (SPAD) from Micro Photon Devices. ${ }^{39}$ In ADTTES-F/Pn-R-F8 composites, the transient PL data were collected separately from the emission wavelength regions dominated by ADT-TES-F donor and by exciplex (charge transfer exciton) using 650SP and 680LP filters from Chroma Tech., respectively. PL lifetime decays were fit with a singleexponential function $\left(a_{0}+a_{1} \exp [-t / \tau]\right)$ to obtain the PL lifetime $\tau$. For electric field-dependent PL measurements, voltage $(V)$ was applied using a Keithley 237 source-measure unit. Spectra were taken at voltages in the $0-200 \mathrm{~V}$ range. The average electric field $\mathrm{E}$ was calculated by using $E=V / L$ where $L$ is the gap between electrodes.

2.4. Photocurrent Measurements. Transient photocurrents were excited by the $355 \mathrm{~nm}, 500$ ps pulsed laser (44.6 kHz, Q-switched, frequency-tripled Nd:YAG from Nanolase, Inc.) at $0.4 \mu \mathrm{J} / \mathrm{cm}^{2}$ incident from the substrate side of the sample. The Keithley 237 source-measure unit provided the bias voltage, and photocurrents amplified by a Centellax UAOL6032VM broadband amplifier were collected by a 50 $\mathrm{GHz}$ digital sampling oscilloscope (DSO) (CSA8200/Tektronix $80 \mathrm{E} 01$ ). Resolution of the set up was $\sim 0.6 \mathrm{~ns}$, limited by the laser pulse width and jitter.

Continuous wave $(\mathrm{cw})$ photocurrents were excited with a $532 \mathrm{~nm}$ laser (frequency-doubled $\mathrm{Nd}: \mathrm{YVO}_{4}$ from Coherent, Inc.) incident from the substrate side of the samples. Currents in the dark and under illumination were collected as a function of applied voltage using a Keithley 237 source-measure unit, and the photocurrents were calculated by subtracting dark currents from the corresponding currents under photoexcitation. In order to observe the long time scale $\mathrm{cw}$ photocurrent dynamics, the voltage was applied to the sample and, after the dark current stabilized, the light was turned on with a shutter (Thorlabs SH05) and turned off after about $80 \mathrm{~s}$, while recording the current as a function of time with the Keithley 237 source-measure unit.

All the experimental data were collected at room temperature.

\section{RESULTS}

3.1. Optical and PL Properties. In all D/A films under study, the absorption spectra were dominated by that of the ADT-TES-F donor (Figure S1a), and contributions of absorption by acceptors to the overall absorption spectra were below the noise level. In all films, optical density at the wavelength of absorption maximum $(\sim 552 \mathrm{~nm}$, Table 1$)$ was between 0.15 and 0.2 , depending on the film.

3.1.1. ADT-TES-F/ADT-R-F Films. Figure $2 \mathrm{a}, \mathrm{b}$ shows $\mathrm{PL}$ spectra of ADT-TES-F/ADT-TIPS-F and ADT-TES-F/ADTTSBS-F films, respectively, normalized at the peak PL values, at several acceptor concentrations. At all concentrations, the PL emission was dominated by that of the ADT-TES-F donor. ${ }^{53}$ The PL intensity increased as the concentration of the ADT-R$\mathrm{F}$ acceptor increased (Figure 2c). The increase in the PL emission (e.g., by a factor of $\sim 3$ and $\sim 6$ at $10 \%$ concentration of ADT-TIPS-F and ADT-TSBS-F, respectively, as compared to a pristine ADT-TES-F film) was accompanied by an increase 

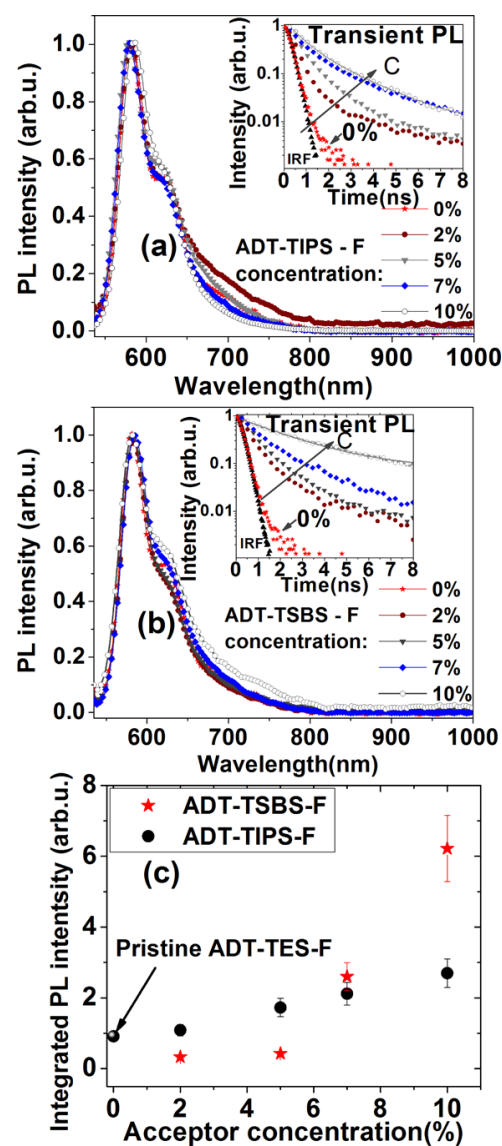

Figure 2. Normalized PL spectra of (a) ADT-TES-F/ADT-TIPS-F and (b) ADT-TES-F/ADT-TSBS-F films, at various acceptor concentrations (C). Insets show PL lifetime decays of the corresponding samples, with fits to the data at $10 \%$ acceptor concentration (solid lines) included. IRF and the PL lifetime decay in a pristine ADT-TES-F film (0\%) are also shown. (c) Integrated PL intensity in ADT-TES-F/ADT-TIPS-F and ADT-TES-F/ADT-TSBS$F$ films as a function of acceptor concentration.

in PL lifetimes as shown in the insets of Figure 2a,b. It has been shown that the PL lifetimes of noninteracting ADT-TES-F molecules are $\sim 10-13 \mathrm{~ns}$, depending on the local environment. $^{39,52}$ In pristine ADT-TES-F films, the PL lifetimes are reduced to $<1-3 \mathrm{~ns}$, dependent on film morphology, ${ }^{18,39,52}$ due to thermally activated exciton diffusion which promotes nonradiative recombination. ${ }^{51}$ For example, PL lifetimes of $<0.4 \mathrm{~ns}$, limited by the instrument response function (IRF), were obtained in a pristine ADT-TES-F film prepared as described in Section 2.2. ${ }^{51}$ Upon ADT-R-F acceptor addition, the PL lifetimes increased, reaching $\sim 3 \mathrm{~ns}(\sim 1.2 \mathrm{~ns})$ in the ADT-TES-F/ADT-TSBS-F (ADT-TES-F/ADT-TIPS-F) composites with $10 \%$ acceptor concentration, as shown in the inset of Figure 2b) (Figure 2a). Observations in Figure 2 suggest that in ADT-TES-F/ADT-R-F films, the ADT-R-F acceptor domains induce disorder in the ADT-TES-F donor domains. This promotes localization of the ADT-TES-F emissive exciton which then exhibits PL properties closer to those of noninteracting ADT-TES-F molecules. The stronger effect in the case of the ADT-TSBS-F acceptor (Figure 2c) at concentrations of above 5\% (at which large acceptor domains formed, Figure S2), which was not observed at lower acceptor concentrations, then must be related to a $1 \mathrm{D}$ "sandwichherringbone" packing motif of the ADT-TSBS-F, which appears to be more disruptive to the $2 \mathrm{D}$ "brick-work" structure of the ADT-TES-F donor domain than the 2D "brick-work" packing of ADT-TIPS-F.

3.1.2. ADT-TES-F/Pn-R-F8 Films. Figure $3 \mathrm{a}, \mathrm{b}$ shows $\mathrm{PL}$ spectra of the ADT-TES-F/Pn-R-F8 composites at various acceptor concentrations. In both systems, exciplex formation was observed, with the emission peak (at $\sim 715 \mathrm{~nm}$ ) closely matching the energy difference between the HOMO of the donor and the LUMO of the acceptor (Table 1). ${ }^{18}$ As the acceptor concentration increased, the exciplex contribution to the overall PL increased, while that of the ADT-TES-F donor decreased. At all acceptor concentrations, the PL quenching of the ADT-TES-F donor was more pronounced in the composites with the Pn-TIPS-F8 acceptor, indicative of a more efficient charge transfer (CT) from ADT-TES-F to PnTIPS-F8 than to Pn-TCHS-F8. Figure 3c shows PL lifetime decays taken from the spectral region of $<650 \mathrm{~nm}$, dominated by the residual PL emission from the ADT-TES-F donor, and from the spectral region of $>680 \mathrm{~nm}$, dominated by the exciplex emission, in ADT-TES-F/Pn-R-F8 films at $10 \%$ acceptor concentration. The ADT-TES-F/Pn-TIPS-F8 exciplex had a longer PL lifetime ( $3.2 \mathrm{~ns})$ than the ADT-TES-F/Pn-TCHSF8 exciplex $(\sim 2.2 \mathrm{~ns})$, and was less prone to dissociation under applied electric field (E-field) (Figure S3), consistent with previous findings in drop-cast films. ${ }^{18}$ More efficient $\mathrm{CT}$ and a longer-lived and less-dissociative exciplex in ADT-TES-F/PnTIPS-F8 films have been attributed to a smaller $\mathrm{D} / \mathrm{A}$ separation in these composites, as compared to that in ADT-TES-F/PnTCHS-F8 films, due to the TIPS group being considerably smaller than the TCHS (Figure 1 and Table 1). ${ }^{18}$ The PL lifetime decay of the residual ADT-TES-F emission in ADTTES-F/Pn-R-F8 films (e.g., lifetime of $\sim 0.5-0.6$ ns at $10 \%$ PnR-F8 acceptor concentration in Figure 3c) was slower than that in pristine ADT-TES-F films $(<0.4 \mathrm{~ns}$ in Figure $3 \mathrm{c})$, but significantly faster than that in ADT-TES-F/ADT-R-F films at comparable concentrations of the acceptor (Section 3.1.1, insets of Figure 2a,b). This is consistent with the residual ADTTES-F emission in ADT-TES-F/Pn-R-F8 films originating from ADT-TES-F intradomain regions, away from the D/A interfaces, which have exciton diffusion properties similar to those of pristine ADT-TES-F films and which are only slightly affected by the presence of acceptors.

Figure 3d shows integrated PL intensity of the emission in the $550-650 \mathrm{~nm}$ spectral region (dominated by the residual ADT-TES-F PL) as a function of Pn-R-F8 concentration. As the concentration of Pn-TIPS-F8 (Pn-TCHS-F8) increased from $2 \%$ to $10 \%$, the ADT-TES-F emission contribution to the overall PL emission decreased from $\sim 30 \%(\sim 50 \%)$ to $\sim 2 \%$ $(\sim 17 \%)$, i.e., by a factor of $\sim 15(\sim 3)$. The most dramatic change occurred between $7 \%$ and $10 \%$ of Pn-R-F8 for both Pn derivatives, especially pronounced in the ADT-TES-F/PnTIPS-F8 films. This is most likely due to differences in the PnR-F8 acceptor domain formation in the case of Pn-TIPS-F8 (2D "brick-work") and Pn-TCHS-F8 (1D "sandwich-herringbone"). It suggests that in ADT-TES-F/Pn-R-F8 composites at acceptor concentrations at which large acceptor domains are present (Figure S2), the overall area of the D/A interface is larger when both donor and acceptor molecules exhibit a $2 \mathrm{D}$ packing motif, as in ADT-TES-F/Pn-TIPS-F8, as compared to a combination of $2 \mathrm{D}$ and $1 \mathrm{D}$ packing motifs in the case of ADT-TES-F/Pn-TCHS-F8.

3.2. Photoconductive Properties. 3.2.1. Transient Photocurrents. Upon photoexcitation with $355 \mathrm{~nm} 500$ ps pulses, 

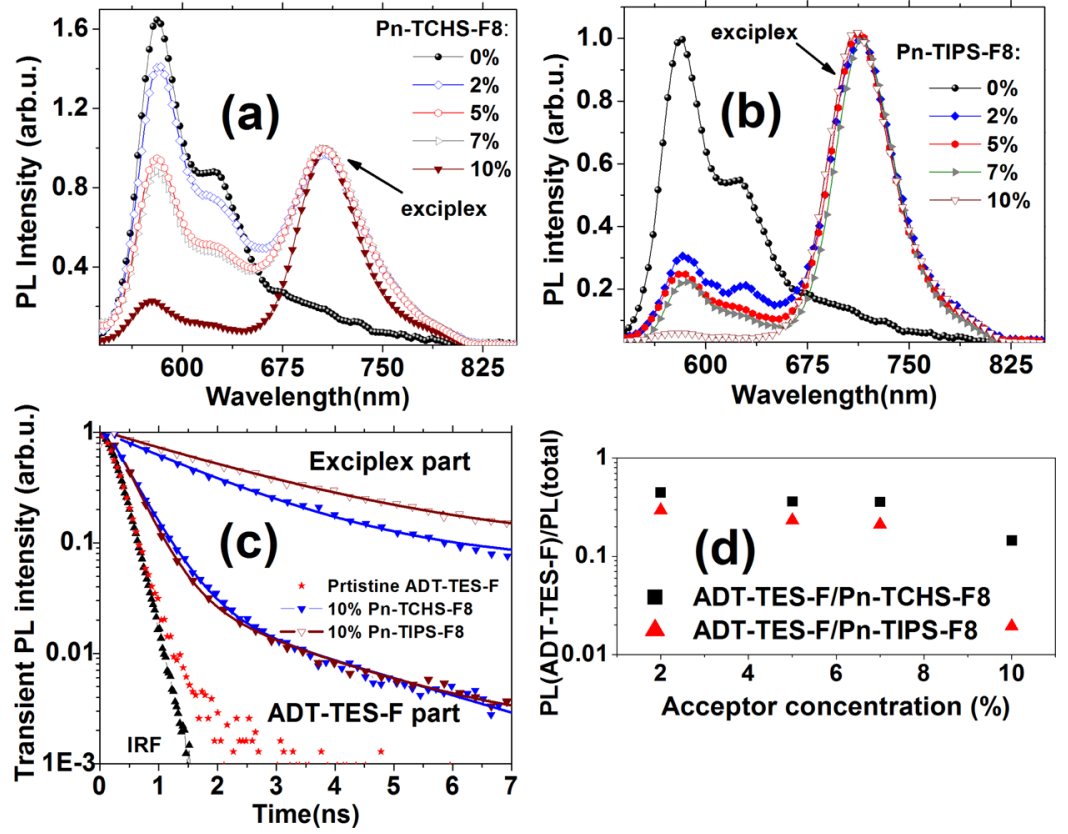

Figure 3. PL spectra of (a) ADT-TES-F/Pn-TCHS-F8 and (b) ADT-TES-F/Pn-TIPS-F8 films, at various acceptor concentrations, normalized at the peak of exciplex emission. The PL spectrum of a pristine ADT-TES-F film is also included. (c) PL lifetime decays of emission in the spectral regions of $<650 \mathrm{~nm}$ (dominated by the residual ADT-TES-F emission) and $>680 \mathrm{~nm}$ (dominated by the exciplex emission) in ADT-TES-F/Pn-TIPS-F8 and ADT-TES-F/Pn-TCHS-F8 films with 10\% acceptor concentration, with fits to the composites data (solid lines) included. The IRF and PL lifetime decay obtained in a pristine ADT-TES-F film are also shown. (d) Integrated PL intensity of emission in the spectral region of $<650 \mathrm{~nm}$, dominated by the residual ADT-TES-F emission, in ADT-TES-F/Pn-TIPS-F8 and ADT-TES-F/Pn-TCHS-F8 films as a function of acceptor concentration.

fast photocurrents were observed in pristine ADT-TES-F films and D/A samples with acceptor concentrations of below $10 \%$. Figure $4 \mathrm{a}, \mathrm{b}$ show transient photocurrent dynamics in pristine ADT-TES-F and several D/A films with 5\% acceptor concentration. In all samples, the rise time (defined here as the time during which the photocurrent increases from $10 \%$ to $90 \%$ of its peak value) of the photocurrents was below $0.6 \mathrm{~ns}$, limited by the excitation laser pulse width and jitter, indicative of fast charge photogeneration, which is consistent with previous observations. ${ }^{18,39,51,58,59}$ At all values of applied Efield, photocurrents measured in the $\mathrm{D} / \mathrm{A}$ films were lower than those in pristine ADT-TES-F films, regardless of the acceptor (Figure 5). ${ }^{53}$ In ADT-TES-F/ADT-R-F films, no considerable effects on the transient photocurrent amplitude due to the sizes of R-groups or the different packing motifs of ADT-R-F acceptors were observed.

At lower acceptor concentrations (below 7\%), the composites with Pn-R-F8 acceptors exhibited photocurrents with higher amplitudes than those in the ADT-TES-F/ADT-RF films (e.g., insets of Figure 4a,b). As the transient photocurrent amplitudes are determined by the product of fast photogeneration efficiency and a sum of hole and electron charge carrier mobilities (in our films dominated by the hole mobility), ${ }^{17,39,51}$ higher peak photocurrents in ADT-TES-F/PnR-F8 films may suggest that the decreased hole mobility due to the disruption of the ADT-TES-F donor crystalline domains by acceptor molecules was partially compensated for by an increased charge photogeneration. In ADT-TES-F/Pn-R-F8 films, such an increase in charge photogeneration efficiency at ns time scales is most likely due to E-field-induced dissociation of the ADT-TES-F/Pn-R-F8 exciplex (Figure S3). ${ }^{17,18,51,60}$ However, at $7 \%$ acceptor concentration, the photocurrents in ADT-TES-F/Pn-R-F8 films were lower than those in ADTTES-F/ADT-R-F films (Figure 5), and the photocurrent
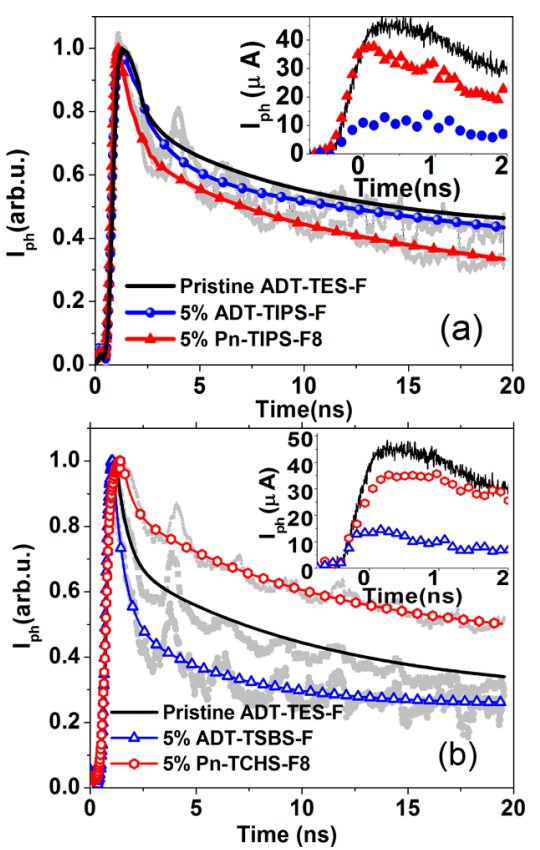

Figure 4. Normalized transient photocurrents obtained in (a) ADTTES-F/ADT-TIPS-F 5\% and ADT-TES-F/Pn-TIPS-F8 5\% films and (b) ADT-TES-F/ADT-TSBS-F 5\% and ADT-TES-F/ADT-Pn-TCHSF8 5\% films under a $355 \mathrm{~nm} 500$ ps photoexcitation at an applied electric field of $40 \mathrm{kV} / \mathrm{cm}$. Data for a pristine ADT-TES-F film are also included. Smooth curves provide a guide for the eye. Insets show a magnified view of the short-time-scale dynamics of the corresponding unnormalized photocurrents.

amplitude in the ADT-TES-F/Pn-TCHS-F8 7\% film was a factor of $\sim 2$ higher than that in the ADT-TES-F/Pn-TIPS-F8 $7 \%$ film, most likely due to a more dissociative ADT-TES-F/ 


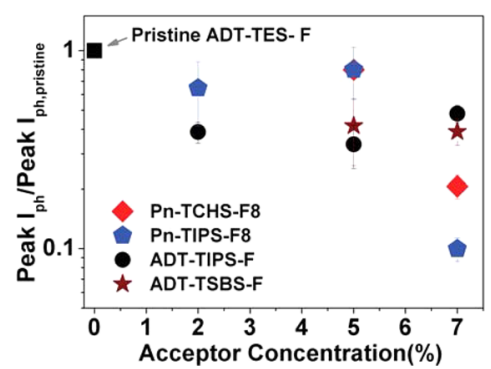

Figure 5. Peak transient photocurrent amplitudes in the D/A films, normalized by that in pristine ADT-TES-F films, as a function of acceptor concentration. The errors bars reflect variations depending on the applied electric field.

Pn-TCHS-F8 exciplex (Figure S3), as discussed in Section 4. At $10 \%$ acceptor concentration, the photocurrents in all $\mathrm{D} / \mathrm{A}$ materials studied were below our minimum detectable signal level, i.e., more than an order of magnitude lower than those in pristine ADT-TES-F films under similar excitation conditions.

The insets of Figures 4a,b show an expanded view of photocurrent dynamics within the first $\sim 2$ ns after photoexcitation in a pristine ADT-TES-F film and in D/A composites with acceptors that exhibit $1 \mathrm{D}$ "sandwich-herringbone" and 2D "brick-work" packing motifs, respectively, at 5\% acceptor concentrations. At these time scales, no effects could be unequivocally attributed to the acceptor packing motif. A significantly faster initial decay of the photocurrent was observed in the composite with the ADT-TSBS-F acceptor (inset of Figure 4a), as compared to that in the composite with the ADT-TIPS-F acceptor (inset of Figure 4b). This could be due to deep charge traps formed at the ADT-TES-F/ADTTSBS-F interfaces, such that the holes trapped within several nanoseconds after photoexcitation do not contribute to the photocurrent for at least $\sim 20 \mathrm{~ns}$. Deep traps in the ADT-TESF/ADT-TSBS-F composites could be caused both by a large $\mathrm{D} / \mathrm{A}$ separation (due to large TSBS groups as compared to TIPS, Table 1) and by the 1D packing motif of ADT-TSBS-F (which was determined by the PL measurements in Figure 2c to be considerably disruptive to the $2 \mathrm{D}$ packing of the $\mathrm{ADT}$ TES-F donor).

The differences in fast photocurrent dynamics between the D/A films with Pn-TIPS-F8 and Pn-TCHS-F8 acceptors have been previously attributed to differences in the contributions of exciplex dissociation to fast charge carrier generation. ${ }^{18}$ In particular, the contribution of the ADT-TES-F/Pn-TCHS-F8 exciplex to charge photogeneration introduced an additional, slower component in the photocurrent rise dynamics (inset of Figure $4 \mathrm{~b}) .{ }^{60}$ This effect was much less pronounced in the ADT-TES-F/Pn-TIPS-F8 films, in which the exciplex is more tightly bound, and the photocurrent rise dynamics was dominated by that of the ADT-TES-F donor (Section 4). ${ }^{17,18}$ The considerably slower photocurrent decay in the composite with a Pn-TCHS-F8 acceptor as compared to that with a PnTIPS-F8 acceptor (Figure 4) is due to reduced recombination of free holes with electrons trapped on Pn-TCHS-F8 molecules (caused by a larger D/A separation in the ADT-TES-F/PnTCHS-F8 film), which was shown by numerical simulations to considerably affect the decay dynamics. ${ }^{17}$ This is consistent with previous experimental results observed in drop-cast films. ${ }^{18}$

3.2.2. Cw Photocurrent. Steady-state photocurrents obtained under $532 \mathrm{~nm} \mathrm{cw}$ photoexcitation of D/A composites, similar to transient photocurrent amplitudes, decreased as the acceptor concentration increased regardless of the acceptor. However, a quantitative comparison of concentration dependencies of $\mathrm{cw}$ photocurrents in various $\mathrm{D} / \mathrm{A}$ films was not possible due to multiple factors affecting the $\mathrm{cw}$ photocurrent amplitudes, including efficiency of charge injection from electrodes, both short and long time-scale charge photogeneration processes, charge carrier mobilities, and charge carrier lifetimes, ${ }^{39,58,61}$ all of which are dependent upon acceptor concentration. For example, the cw photocurrents in $\mathrm{D} / \mathrm{A}$ films at $10 \%$ acceptor concentrations were a factor of $4-$ 10 lower than those in pristine ADT-TES-F films, depending more on the sample and on the applied E-field than on the nature of the acceptor. One common feature shared by all $\mathrm{D} / \mathrm{A}$ composites studied, however, was that the concentration dependence of the $\mathrm{cw}$ photocurrent closely followed that of the dark current, which suggests that the decrease of the $\mathrm{cw}$ photocurrent upon acceptor addition was mostly unrelated to charge photogeneration. For example, in ADT-TES-F/ADTTIPS-F films, as the ADT-TIPS-F concentration increased from $2 \%$ to $10 \%$, both the dark current and the cw photocurrent decreased by a factor of $\sim 4$, most likely due to a lower hole mobility caused by an increased disorder (inset of Figure 6a).

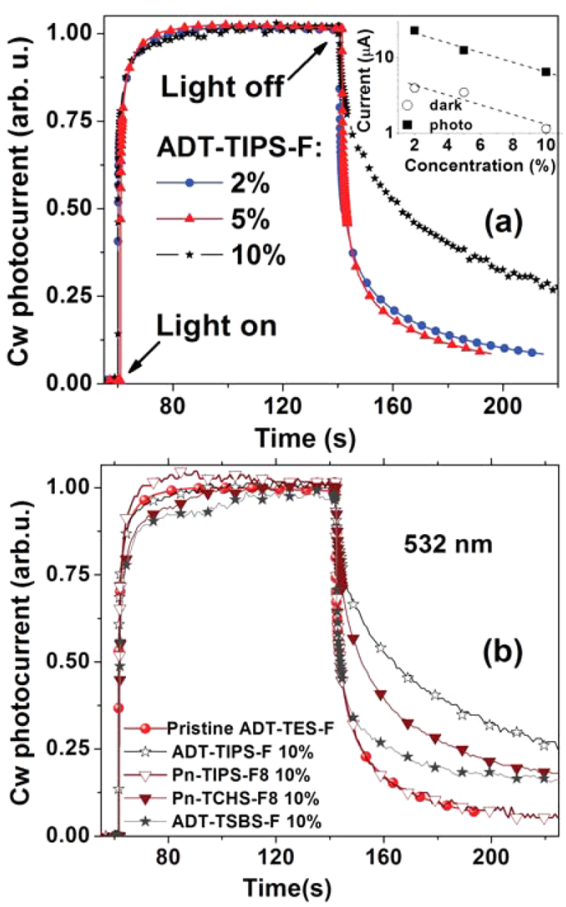

Figure 6. Dynamics of normalized cw photocurrents in (a) ADT-TESF/ADT-TIPS-F films at several acceptor concentrations and (b) various $\mathrm{D} / \mathrm{A}$ films at $10 \%$ acceptor concentration. Dynamics in a pristine ADT-TES-F film are also included. At time $t=0$, an electric field of $20 \mathrm{kV} / \mathrm{cm}$ is applied, and at $t=60(140) \mathrm{s}$, the $532 \mathrm{~nm} 10$ $\mathrm{mW} / \mathrm{cm}^{2} \mathrm{cw}$ light is turned on (off). Inset in (a) shows dark and $\mathrm{cw}$ photocurrents in ADT-TES-F/ADT-TIPS-F films as a function of ADT-TIPS-F concentration.

Additional evidence of the increased disorder at $10 \% \mathrm{ADT}$ TIPS-F concentration can be seen in Figure 6a, which shows the $\mathrm{cw}$ photocurrent dynamics for ADT-TES-F/ADT-TIPS-F films at several ADT-TIPS-F concentrations. At $10 \%$ of $\mathrm{ADT}$ TIPS-F, the $\mathrm{cw}$ photocurrent decay after the light was turned off was considerably slower than that at lower acceptor 
concentrations, indicative of a large density of shallow charge traps. In the composite with $10 \%$ of ADT-TSBS-F (Figure $6 \mathrm{~b}$ ), faster $\mathrm{cw}$ photocurrent decay (as compared to that in the composite with ADT-TIPS-F) suggests deeper traps in the ADT-TES-F/ADT-TSBS-F films, consistent with the observations at ns time scales (Figure 4). In the ADT-TES-F/PnTCHS-F8 10\% film, cw photocurrent decay dynamics were slower than those in the composite with the Pn-TIPS-F8 acceptor due to slower trap-assisted recombination, also consistent with the observations at the nanoseconds timescales (Figure 4). ${ }^{17}$

\section{DISCUSSION}

PL measurements in ADT-TES-F/ADT-R-F films (Section 3.1.1) revealed contributions solely due to film structure and morphology changes caused by addition of an acceptor to the ADT-TES-F donor exciton dynamics. In particular, as the ADT-TES-F donor domains were more disrupted by the formation of acceptor domains, the emissive ADT-TES-F exciton became longer lived and more emissive. However, similar to previous findings in various ADT-TES-F-based films, ${ }^{18,51}$ the emissive ADT-TES-F exciton in films prepared as described in Section 2.2 did not appear to significantly contribute to nanoseconds time-scale charge photogeneration, and the photocurrents in ADT-TES-F/ADT-TSBS-F or ADTTES-F/ADT-TIPS-F films were lower than those in pristine ADT-TES-F films.

In ADT-TES-F/Pn-R-F8 films, properties of the emissive exciplex and the amount of ADT-TES-F donor PL quenching provided a sensitive measure of the $\mathrm{D} / \mathrm{A}$ charge transfer and changes in the $\mathrm{D} / \mathrm{A}$ interfacial area upon acceptor domain formation (Section 3.1.2). Previous studies showed that exciplex dissociation in ADT-TES-F-based D/A materials contributed to charge photogeneration, ${ }^{18,51,60}$ although this contribution was relatively small. For example, numerical simulations revealed that only $\sim 10 \%$ of all charge carriers photogenerated at time-scales of up to $\sim 20$ ns after sub-ns pulsed photoexcitation of ADT-TES-F/Pn-TIPS-F8 2 wt \% drop-cast films were due to exciplex dissociation, whereas the rest of the charge carriers were generated in the ADT-TES-F donor. ${ }^{17}$ (This is in contrast to ADT-TES-F/PCBM 2 wt \% films, in which about $50 \%$ of photogenerated carriers were due to $\mathrm{CT}$ exciton dissociation under similar experimental conditions.) ${ }^{17}$ At higher acceptor concentrations, i.e., when the exciplex population is large, one would expect an increased contribution of the exciplex dissociation to charge photogeneration. However, it has been shown by numerical simulations of transient photocurrents that introduction of Pn-R-F8 molecules into ADT-TES-F films lowers the overall charge photogeneration efficiency (due to decreased charge photogeneration in the ADT-TES-F donor) and lowers charge carrier mobilities, both of which contribute to a reduction in the transient photocurrent amplitude. ${ }^{17}$ Thus, at higher acceptor concentrations, an increase in charge photogeneration in ADTTES-F/Pn-R-F8 films due to exciplex dissociation was not large enough to compensate for a decrease in the charge generation in the ADT-TES-F donor and for detrimental changes in charge transport properties (caused by an increase in donor domain disorder with acceptor concentration), which resulted in lower photocurrents.

A weaker acceptor concentration dependence of the $\mathrm{cw}$ photocurrent (Section 3.2.2) as compared to that of the transient photocurrent amplitude (Figure 5) is most likely related to charge carrier lifetimes, which in most D/A composites with higher acceptor loading are longer than in pristine ADT-TES-F films (Figure 6b). ${ }^{18,60,62}$ This is due to a disorder-induced increase in charge trapping as well as slow recombination of trapped electrons with mobile holes in the $\mathrm{D} /$ A films as compared to pristine donor films (Figure 6b). ${ }^{17}$ Longer carrier lifetimes in the D/A films manifest, for example, in slower $\mathrm{cw}$ photocurrent decays in $\mathrm{D} / \mathrm{A}$ composites as compared to those in pristine ADT-TES-F donor films. As the cW photocurrent amplitudes are proportional to carrier lifetimes, ${ }^{63}$ longer carrier lifetimes enhance the $\mathrm{cw}$ photocurrents $^{18,39}$ but do not significantly contribute to the fast photocurrent amplitudes in Figure 5.

It has been previously established that solid-state packing of functionalized ADT and Pn derivatives, determined by side groups $\mathrm{R}$ (Figure 1), dramatically affects optical and electronic properties of $\mathrm{ADT}$ and Pn films. ${ }^{39,55}$ For example, derivatives with 2D "brick-work" packing, such as ADT-TES-F, exhibited much better performance in solution-deposited TFTs than those with other packing motifs such as 1D "slip-stack". 55 This is largely due to the high-mobility $a-b$ plane of the crystallites being coincident with the plane of the substrate and the $2 \mathrm{D} \pi$ stacking being conducive to formation of large-area crystallites. $^{43,47}$ In contrast, most successful Pn derivatives in BHJ solar cells with a polymer donor and functionalized Pn acceptor $^{22}$ were those with a 1D "sandwich-herringbone" packing, such as Pn-TCHS-F8 used in our studies. This is because this geometry resulted in an acceptor domain structure more favorable for charge generation, one that produced a large $\mathrm{D} / \mathrm{A}$ interfacial area. In our small-molecule D/A composites at higher acceptor loading, the $\mathrm{D} / \mathrm{A}$ interfacial area was also affected by the acceptor packing motif (Section 3.1.2). In particular, a larger D/A interfacial area was obtained in the composites with the Pn-TIPS-F8 acceptor, which exhibits a 2D "brick-work" packing similar to that of the ADT-TES-F donor, than in those with the Pn-TCHS-F8 acceptor. Nevertheless, our experiments showed no particular advantage for the photoconductive properties of small-molecule $\mathrm{BHJ}$ s in either packing motif studied here, at least in the planar geometry that takes advantage of the high charge carrier mobilities in the $a-b$ plane of the crystallites. However, this could change if the CT excitons formed at the $\mathrm{D} / \mathrm{A}$ interfaces were more dissociative, thus contributing more significantly to charge photogeneration, than the tightly bound emissive exciplexes observed here. This could be achieved by, for example, further increasing the D/A LUMO offset, D/A separation at the $\mathrm{D} / \mathrm{A}$ interfaces, or both. ${ }^{11,18,64}$ Then, the advantage of a larger $\mathrm{D} / \mathrm{A}$ area, observed in the ADT-TES-F/Pn-TIPS-F8 composites with both $\mathrm{D}$ and $\mathrm{A}$ packing in the $2 \mathrm{D}$ "brick-work" structures, could bring an enhancement in the photocurrent.

\section{SUMMARY}

Based on the results of our experiments, we assigned the following observations to the effects of the D/A LUMO offset, $\mathrm{D} / \mathrm{A}$ separation at the $\mathrm{D} / \mathrm{A}$ interfaces, and solid-state packing.

Effects of D/A LUMO Offset. The main effect of the D/A LUMO offset on the optoelectronic properties of our smallmolecule D/A materials was exciplex formation in ADT-TESF/Pn-R-F8 films (Figure 3). Exciplex dissociation contributed to charge carrier photogeneration at nanoseconds time-scales, leading to higher transient photocurrent amplitudes in ADTTES-F/Pn-R-F8 films than in ADT-TES-F/ADT-R-F films at low $(<7 \%)$ acceptor concentrations (Figure 4). However, at 
higher acceptor concentrations, the D/A LUMO offset of $\sim 0.55 \mathrm{eV}$ in ADT-TES-F/Pn-R-F8 films was not sufficient to yield higher photocurrents than those in ADT-TES-F/ADT-RF films with the D/A LUMO offset close to zero.

Effects of D/A Separation. The D/A separation considerably affected CT, exciplex emission, and E-fieldinduced exciplex dissociation properties in ADT-TES-F/Pn-RF8 films, with larger D/A separation corresponding to less efficient CT and a shorter-lived and more dissociative exciplex (Figure 3). In the same films, transient photocurrent rise and decay dynamics were also dependent on the D/A separation due to varying contributions of exciplex dissociation to charge photogeneration and differences in trap-assisted charge recombination, respectively (Figure 4). In ADT-TES-F/ADTR-F composites, the D/A separation affected charge trapping properties observed via transient and $\mathrm{cw}$ photocurrent decay dynamics (Figures 4 and $6 \mathrm{~b}$ ).

Effects of Solid-State Packing. Solid-state packing affected the formation of D/A interfaces in ADT-TES-F/PnR-F8 films, with a D/A interfacial area increasing faster with acceptor concentration in the case of Pn-TIPS-F8 acceptor than in Pn-TCHS-F8 (Figure 3d). In ADT-TES-F/ADT-R-F films, differences in acceptor domain structures resulted in differences in the emissive ADT-TES-F exciton properties (manifest in the PL lifetimes and emission intensities, Figure 2) and in disorder (observed in the cw photocurrent dynamics, Figure 6).

In conclusion, we investigated the dependence of the PL and photocurrent on the D/A LUMO offset, D/A separation, and acceptor solid-state packing. Addition of an ADT-R-F acceptor to an ADT-TES-F donor introduced disorder which resulted in increased PL emission and in reduced photocurrents. In ADTTES-F/Pn-R-F8 films, CT from ADT-TES-F to Pn-R-F8 was observed via formation of an exciplex that contributed to charge carrier photogeneration. A larger $\mathrm{D} / \mathrm{A}$ interfacial area was observed in the ADT-TES-F/Pn-TIPS-F8 films, in which both the donor and acceptor exhibited 2D "brick-work" packing, as compared to ADT-TES-F/Pn-TCHS-F8, in which the acceptor assumed 1D "sandwich-herringbone" packing. However, addition of Pn-R-F8 acceptors at higher concentrations was detrimental to the photocurrent, regardless of the R-groups and packing motifs, due to lower charge photogeneration efficiency in the ADT-TES-F donor and due to a high degree of disorder that lowered charge carrier mobility. Higher D/A offsets and/or larger D/A separation at the D/A interface would be necessary to improve CT exciton dissociation efficiency and enhance charge photogeneration sufficiently to compensate for the detrimental changes in charge transport properties upon an increase in acceptor concentration.

\section{ASSOCIATED CONTENT}

\section{(S Supporting Information}

Figure S1: absorption and PL of pristine ADT-R-F and Pn-RF8 films. Figure S2: optical images of select films. Figure S3: PL spectra of ADT-TES-F/Pn-R-F8 10\% films at various voltages. This information is available free of charge via the Internet at http://pubs.acs.org.

\section{AUTHOR INFORMATION}

\section{Corresponding Author}

*E-mail: oksana@science.oregonstate.edu.

Notes

The authors declare no competing financial interest.

\section{ACKNOWLEDGMENTS}

We thank J. Ward and Prof. O. D. Jurchescu for helpful suggestions regarding PFBT treatment of substrates. This work was supported by the NSF grant DMR 1207309. J.E.A. thanks the NSF (Grant DMR 1035257) for support for the synthesis of the materials used in this study.

\section{REFERENCES}

(1) Heeger, A. J. Chem. Soc. Rev. 2010, 39, 2354-2371.

(2) Ostroverkhova, O., Ed. Handbook of Organic Materials for Optical and (Opto)Electronic Devices, 1st ed.; Woodhead Publishing Limited: Cambridge, U.K., 2013.

(3) Samuel, I. D. W.; Turnbull, G. A. Chem. Rev. 2007, 107, 12721295.

(4) Katz, H. E.; Huang, J. Annu. Rev. Mater. Res. 2009, 39, 71-92.

(5) Yeh, N.; Yeh, P. Renewable Sustainable Energy Rev. 2013, 21, 421-431.

(6) Ostroverkhova, O.; Moerner, W. E. Chem. Rev. 2004, 104, 32673314.

(7) Facchetti, A. Chem. Mater. 2011, 23, 733-758.

(8) Sliaužys, G.; Arlauskas, K.; Gulbinas, V. Phys. Status Solidi A 2012, 209, 1302-1306.

(9) Schwenn, P. E.; Gui, K.; Zhang, Y.; Burn, P. L.; Meredith, P.; Powell, B. J. Org. Electron. 2012, 13, 2538-2545.

(10) Etzold, F.; Howard, I. A.; Mauer, R.; Meister, M.; Kim, T.-D.; Lee, K.-S.; Baek, N. S.; Laquai, F. J. Am. Chem. Soc. 2011, 133, 94699479.

(11) Clarke, T. M.; Durrant, J. R. Chem. Rev. 2010, 110, 6736-6767. (12) Sun, Y.; Welch, G. C.; Leong, W. L.; Takacs, C. J.; Bazan, G. C.; Heeger, A. J. Nat. Mater. 2011, 11, 44-48.

(13) Walker, B.; Kim, C.; Nguyen, T.-Q. Chem. Mater. 2011, 23, 470-482.

(14) Kaake, L. G.; Jasieniak, J. J.; Bakus, R. C.; Welch, G. C.; Moses, D.; Bazan, G. C.; Heeger, A. J. J. Am. Chem. Soc. 2012, 134, 1982819838 .

(15) Credgington, D.; Jamieson, F. C.; Walker, B.; Nguyen, T.-Q.; Durrant, J. R. Adv. Mater. 2012, 24, 2135-2141.

(16) Lin, Y.; Li, Y.; Zhan, X. Chem. Soc. Rev. 2012, 41, 4245-4272.

(17) Johnson, B.; Kendrick, M.; Ostroverkhova, O. J. Appl. Phys. 2013, 114, 094508.

(18) Kendrick, M. J.; Neunzert, A.; Payne, M. M.; Purushothaman, B.; Rose, B. D.; Anthony, J. E.; Haley, M. M.; Ostroverkhova, O. J. Phys. Chem. C 2012, 116, 18108-18116.

(19) Bakulin, A. A.; Dimitrov, S. D.; Rao, A.; Chow, P. C. Y.; Nielsen, C. B.; Schroeder, B. C.; McCulloch, I.; Bakker, H. J.; Durrant, J. R.; Friend, R. H. J. Phys. Chem. Lett. 2013, 4, 209-215.

(20) Ohkita, H.; Cook, S.; Astuti, Y.; Duffy, W.; Tierney, S.; Zhang, W.; Heeney, M.; McCulloch, I.; Nelson, J.; Bradley, D. D. C.; Durrant, J. R. J. Am. Chem. Soc. 2008, 130, 3030-3042.

(21) Holcombe, T. W.; Norton, J. E.; Rivnay, J.; Woo, C. H.; Goris, L.; Piliego, C.; Griffini, G.; Sellinger, A.; Brédas, J.-L.; Salleo, A.; Fréchet, J. M. J. J. Am. Chem. Soc. 2011, 133, 12106-12114.

(22) Lim, Y.-F.; Shu, Y.; Parkin, S. R.; Anthony, J. E.; Malliaras, G. G. J. Mater. Chem. 2009, 19, 3049-3056.

(23) Dimitrov, S. D.; Bakulin, A. A.; Nielsen, C. B.; Schroeder, B. C.; Du, J.; Bronstein, H.; McCulloch, I.; Friend, R. H.; Durrant, J. R. J. Am. Chem. Soc. 2012, 134, 18189-18192.

(24) Zhang, W.; Wang, Y.-W.; Hu, R.; Fu, L.-M.; Ai, X.-C.; Zhang, J.P.; Hou, J.-H. J. Phys. Chem. C 2013, 117, 735-749.

(25) Ren, G.; Schlenker, C. W.; Ahmed, E.; Subramaniyan, S.; Olthof, S.; Kahn, A.; Ginger, D. S.; Jenekhe, S. A. Adv. Funct. Mater. 2013, 23, $1238-1249$

(26) Murthy, D. H. K.; Gao, M.; Vermeulen, M. J. W.; Siebbeles, L. D. A.; Savenije, T. J. J. Phys. Chem. C 2012, 116, 9214-9220.

(27) Opitz, A.; Wagner, J.; Brütting, W.; Salzmann, I.; Koch, N.; Manara, J.; Pflaum, J.; Hinderhofer, A.; Schreiber, F. IEEE J. Sel. Top. Quantum Electron. 2010, 16, 1707-1717. 
(28) Beljonne, D.; Cornil, J.; Muccioli, L.; Zannoni, C.; Brédas, J.-L.; Castet, F. Chem. Mater. 2011, 23, 591-609.

(29) Grancini, G.; Maiuri, M.; Fazzi, D.; Petrozza, A.; Egelhaaf, H.-J.;

Brida, D.; Cerullo, G.; Lanzani, G. Nat. Mater. 2012, 29-33.

(30) Mingebach, M.; Walter, S.; Dyakonov, V.; Deibel, C. Appl. Phys. Lett. 2012, 100, 193302.

(31) Faist, M. A.; Kirchartz, T.; Gong, W.; Ashraf, R. S.; McCulloch, I.; de Mello, J. C.; Ekins-Daukes, N. J.; Bradley, D. D. C.; Nelson, J. J. Am. Chem. Soc. 2012, 134, 685-692.

(32) Askat, E.; Jailaubekov, A. P.; Willard, J.; Tritsch, W.-L. C.; Na, S.; Raluca, G.; Loren, G. K.; Kenrick, J. W.; Kevin, L.; Rossky, P. J. Nat. Mater. 2012, 12, 66-73.

(33) Singh, S.; Pandit, B.; Basel, T. P.; Li, S.; Laird, D.; Vardeny, Z. V. Phys. Rev. B 2012, 85, 205206.

(34) Wang, M.; Wudl, F. J. Mater. Chem. 2012, 22, 24297-24314.

(35) Lyons, B. P.; Clarke, N.; Groves, C. Energy Environ. Sci. 2012, 5, $7657-7663$.

(36) Lam, S. L.; Liu, X.; Zhao, F.; Lee, C.-L. K.; Kwan, W. L. Chem. Commun. 2013, 49, 4543-4545.

(37) Fitzner, R.; Elschner, C.; Weil, M.; Uhrich, C.; Körner, C.; Riede, M.; Leo, K.; Pfeiffer, M.; Reinold, E.; Mena-Osteritz, E.; Bäuerle, P. Adv. Mater. 2012, 24, 675-680.

(38) Gundlach, D. J.; et al. Nat. Mater. 2008, 7, 216-21.

(39) Platt, A. D.; Day, J.; Subramanian, S.; Anthony, J. E.; Ostroverkhova, O. J. Phys. Chem. C 2009, 113, 14006-14014.

(40) Peumans, P.; Forrest, S. R. Chem. Phys. Lett. 2004, 398, 27-31.

(41) Anthony, J. E. Chem. Rev. 2006, 106, 5028-5048.

(42) Subramanian, S.; Park, S. K.; Parkin, S. R.; Podzorov, V.; Jackson, T. N.; Anthony, J. E. J. Am. Chem. Soc. 2008, 130, 27062707.

(43) Goetz, K. P.; Li, Z.; Ward, J. W.; Bougher, C.; Rivnay, J.; Smith, J.; Conrad, B. R.; Parkin, S. R.; Anthopoulos, T. D.; Salleo, A.; Anthony, J. E.; Jurchescu, O. D. Adv. Mater. 2011, 23, 3698-3703.

(44) Swartz, C. R.; Parkin, S. R.; Bullock, J. E.; Anthony, J. E.; Mayer, A. C.; Malliaras, G. G. Org. Lett. 2005, 7, 3163-3166.

(45) Purushothaman, B. Partially Halogenated Acenes and Heteroacenes for Organic Electronics. Ph.D. Thesis, University of Kentucky, Lexington, KY, 2011.

(46) Gundlach, D. J.; et al. Nat. Mater. 2008, 7, 216-21.

(47) Park, S. K.; Mourey, D. A.; Subramanian, S.; Anthony, J. E.; Jackson, T. N. Appl. Phys. Lett. 2008, 93, 043301.

(48) Jurchescu, O. D.; Subramanian, S.; Kline, R. J.; Hudson, S. D.; Anthony, J. E.; Jackson, T. N.; Gundlach, D. J. Chem. Mater. 2008, 20, 6733-6737.

(49) Kline, R. J.; Hudson, S. D.; Zhang, X.; Gundlach, D. J.; Moad, A. J.; Jurchescu, O. D.; Jackson, T. N.; Subramanian, S.; Anthony, J. E.; Toney, M. F.; Richter, L. J. Chem. Mater. 2011, 23, 1194-1203.

(50) Tang, M. L.; Reichardt, A. D.; Wei, P.; Bao, Z. J. Am. Chem. Soc. 2009, 131, 5264-5273.

(51) Platt, A. D.; Kendrick, M. J.; Loth, M.; Anthony, J. E.; Ostroverkhova, O. Phys. Rev. B 2011, 84, 235209.

(52) Shepherd, W. E. B.; Platt, A. D.; Hofer, D.; Ostroverkhova, O.; Loth, M.; Anthony, J. E. Appl. Phys. Lett. 2010, 97, 163303.

(53) Paudel, K.; Johnson, B.; Neunzert, A.; Thieme, M.; Anthony, J.; Ostroverkhova, O. Proc. SPIE Opt. Photonics 2013, 8827-25.

(54) Ostroverkhova, O. In Organic Electronics: Emerging Concepts and Technologies; Cicoria, F., Santato, C., Eds.; Wiley-VCH: Weinheim, Germany, 2013; Chapter 10, pp 233-255.

(55) Anthony, J. E. Chem. Rev. 2006, 106, 5028-5048.

(56) Ostroverkhova, O.; Shcherbyna, S.; Cooke, D. G.; Egerton, R. F.; Hegmann, F. A.; Tykwinski, R. R; Parkin, S. R.; Anthony, J. E. J. Appl. Phys. 2005, 98, 033701.

(57) Kim, C. H.; Hlaing, H.; Carta, F.; Bonnassieux, Y.; Horowitz, G.; Kymissis, I. ACS Appl. Mater. Interfaces 2013, 5, 3716-3721.

(58) Day, J.; Subramanian, S.; Anthony, J. E.; Lu, Z.; Twieg, R. J.; Ostroverkhova, O. J. Appl. Phys. 2008, 103, 123715.

(59) Day, J.; Platt, A. D.; Subramanian, S.; Anthony, J. E.; Ostroverkhova, O. J. Appl. Phys. 2009, 105, 103703.
(60) Shepherd, W. E. B.; Platt, A. D.; Kendrick, M. J.; Loth, M. A.; Anthony, J. E.; Ostroverkhova, O. J. Phys. Chem. Lett. 2011, 2, 362366.

(61) Day, J.; Subramanian, S.; Anthony, J. E.; Lu, Z.; Twieg, R. J.; Ostroverkhova, O. J. Appl. Phys. 2008, 103, 123715.

(62) Day, J.; Platt, A. D.; Ostroverkhova, O.; Subramanian, S.; Anthony, J. E. Appl. Phys. Lett. 2009, 94, 013306.

(63) Moses, D.; Wang, J.; Yu, G.; Heeger, A. J. Phys. Rev. Lett. 1998, $80,2685-2688$.

(64) Deibel, C.; Strobel, T.; Dyakonov, V. Adv. Mater. 2010, 22, 4097-4111. 